DOI https://doi.org/10.30525/978-9934-26-046-9-29

\title{
ANALYSIS OF METHODS OF PROTECTION OF RADIOELECTRONIC EQUIPMENT AGAINST ELECTROMAGNETIC INFLUENCE
}

\author{
Iasechko M. M. \\ Doctor of Technical Sciences, \\ Associate professor at the Department of Air Defense Armaments \\ of the Land Forces \\ Ivan Kozhedub Kharkiv National Air Force University \\ Kharkiv, Ukraine \\ Kozyr A. H. \\ Candidate of Technical Sciences, \\ Chief of Research and Development Section of Armament \\ and Military Equipment Testing and Certification \\ State Scientific Research Institute of Armament \\ and Military Equipment Testing and Certification \\ Chernihiv, Ukraine \\ Bashynskyi K. V. \\ Candidate of Technical Sciences, \\ Head of the Group of the 1285 Military Representatives \\ Ministry of Defense of Ukraine \\ Kyiv, Ukraine
}

Designed to reduce the reflection of electromagnetic radiation inside the shielding objects, as well as to ensure their electrical sealing $[1 ; 4]$.

The following main types of RAC (radar absorbing coatings) are known, the classification of which is shown in figure 1.

The coatings are flat-layered in which a decrease in the incident radiation is achieved by the mutual attenuation of waves reflected from the object's surface and the coating surface. Falling EMR (electromagnetic radiation) is repeatedly reflected from the separation of two "coatingobject» media and is partially absorbed by the coating substance. If the coverage is perfect, then the total field in the direction of the source of the incident wave is zero. An example of such coatings is the quarter-wave absorber EMR. The disadvantages of such coatings are their narrow band and high dependence of the reflection coefficient on the angle of the incidence. 


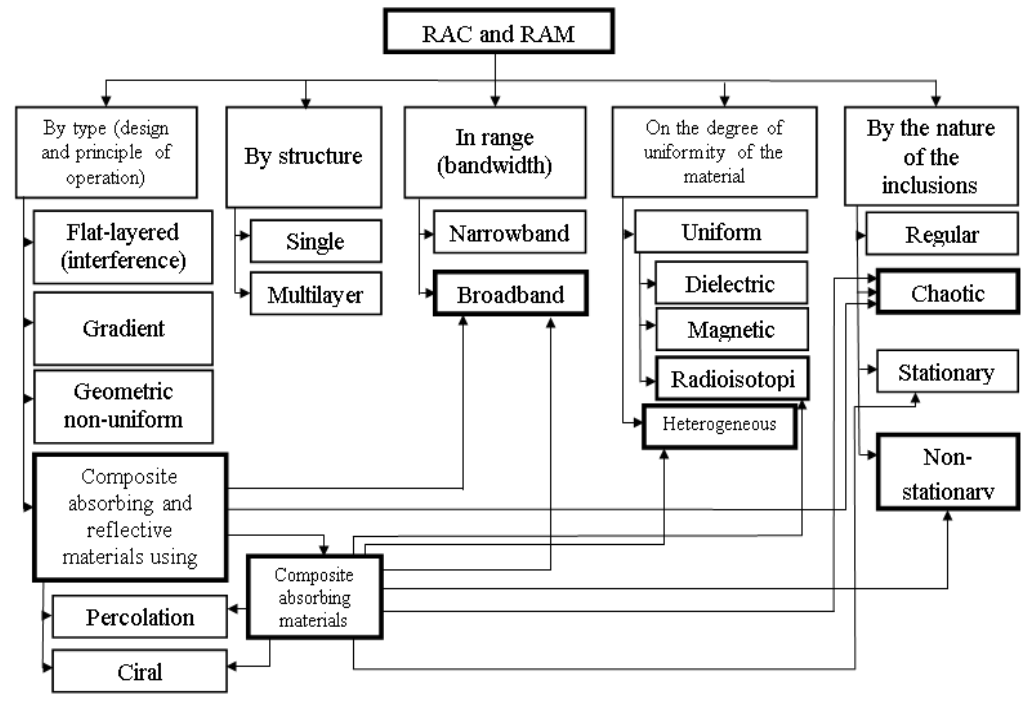

Figure 1. Classification radar absorbing coatings

The expansion of the working strip can be achieved as a result of a multi-layer structures use with a gradual change in the characteristics of the layers. If the number of layers of the multilayered stepped RAC is unlimited increased (in practice it's up to 10), then such a flat layer structure will turn into a gradient type coating.

For the most effective absorption of microwave radiation, it is better to use the coatings and materials with high dielectric and (or) magnetic permeability. The main physical properties of materials (dielectrics, ferro electrics, metals and their oxides, semiconductors, ferrites), which can be used for use in creating EMR absorbing structures, are given in [3].

Gradient type coatings have low reflective properties in a wide frequency band. However, they are bulky and the most difficult from the point of view of practical implementation, which is a disadvantage. Geometrically in-homogeneous RAM may have a different shape: saw-like, cone-like, pyramidal. In gradient coatings the coordination with free space is due to the gradual changes in the properties of the absorbing medium based on the use of the constant parameters layers set. The advantages of coatings of this type include a wide working band with a low reflection coefficient. The disadvantages are their sizes, which significantly exceed the sizes of planar RAC and RAM (radio-electronic means). 
The existing RAM by the mechanism of absorption can be divided into two classes:

1. Dielectric materials with low dielectric constant and high absorption level of electromagnetic energy due to electrical conductivity.

2. Ferromagnetic materials that provide absorption of electromagnetic radiation due to magnetic reversal losses.

The most effective ferromagnetic materials with magnetic losses. However, it should be noted that despite the great achievements in the field of creating special absorbing materials based on ferrite, ceramics and ferrite compounds, it is still not possible to manufacture an effective absorber of electromagnetic radiation EMR in a fairly wide wavelength range at different angles of incidence. Thus, the reflective R of the NZ-31, NZ-41 NZ-51 (Eccosorb) materials as a function of frequency is shown in [3], showing that the reflection ability of these coatings, which are $5 \ldots 8 \mathrm{~mm}$ thick, is 20 $\mathrm{dB}$ (that is, no more than $1 \%$ of the incident energy is reflected) is stored only in the frequency band $=250 \ldots 400 \mathrm{MHz}$ To extend the frequency range using multi-layer coatings. However, the high proportion of ferromagnetic materials makes it unacceptable to use them on the flying aircraft (FA). But in work [3] it is noted that Russian specialists managed to create the world's first protective material of small specific gravity (only 0.2 $\mathrm{kg} / \mathrm{m}^{2}$ ) and with high absorbing properties. The material protects them from electromagnetic radiation EMR in the range from $0.5 \mathrm{GHz}$ to $50 \mathrm{GHz}$, and also reduces the level of electromagnetic exposure by up to $10-30 \mathrm{~dB}$ (radiation reflected from the material) and up to $100 \mathrm{~dB}$ (radiation passed for the material).

Ferrite fabric has the highest flexibility, which allows it to cover almost everything. The material can be used in the temperature range from 50 to +110 degrees Celsius. At present, the production of linen size 500 by 500 millimeters.

To expand the frequency range while ensuring high efficiency of absorption of electromagnetic waves, composite materials with various kinds of inclusions and the so-called artificial media [3-5], whose work is based on the principle of scattering and absorption of electromagnetic radiation EMR at resonant frequencies determined by the size of inclusions. So, to create effective RACs, the possibility of using chiral and percolation materials related to the composite materials [4-4] is being investigated.

Chiral environment is an isotropic dielectric, in which mirrorasymmetric elements are randomly distributed. Percolation materials use disordered mixtures of high and low conductive particles. At a certain critical concentration of highly conductive particles, which is called the 
percolation threshold, a metal-insulator transition is observed in such an environment [4].

Percolation environment have abnormally high dielectric constant. Conductivity is characterized by anomalous non linearity. By changing the conductivity, size and concentration of conductive inclusions, it is possible to vary the capacitive and inductive properties of percolation clusters and, therefore, create materials with the necessary electro-physical properties, including those that have low-reflective properties. An example of coatings made from such materials are coatings that use a powder consisting of miniature ceramic medium coated with metal $[2,4]$. The diameter of the ceramic medium is in the range of 5 ... 75 microns. When using such powder as an impurity to the paint, its cost lies within the range of \$1-2 per square foot $(1$ foot $=0.3048 \mathrm{~m})$. At the same time, it increases the paint weight by just 0.05 pounds per square foot $(1$ pound $=0.48 \mathrm{~kg}$ ) to the weight of the paint. It is argued that, based on the properties of such a powder, coatings can be created to absorb electromagnetic radiation in the range $1 \ldots 100 \mathrm{GHz}$.

Magnetic granular materials are considered to be promising. They represent a structural composite in which ferromagnetic particles with a crystal structure are placed in a dielectric matrix [1-2]. One of the advantages of such materials is the ability to easily control their parameters by changing the concentration of the component introduced into the composite. In this case, there is a real possibility of creating an absorbing material with a low reflection coefficient of high-frequency electromagnetic radiation [5]. Dielectric matrices with ferromagnetic particles included in them are widely used as absorbers [4], as well as as fillers for wave guides and resonators in various non-reciprocal microwave engineering devices. In [6], it was shown that in composites based on hexagonal ferrites (HF) and polymer bonding, the absorption of electromagnetic radiation EMR occurs due to the natural ferromagnetic resonance. Such materials are promising to use in the frequency range $2.6 \mathrm{GHz}$.

The results of the conducted research allow to conclude that RAC and RAM models based on the modified materials have a high degree of a EMR absorption.

\section{References:}

1. Iasechko M., Tymochko O., Shapran Y., Trofymenko I., Maksiuta D., Sytnyk Y. Loss definition of charged particles in the discharge gap of the opening of the box-screens during the formation of a highly conductive channel. IJATCSE. 2019. № 8 (1.3). Pp. 1-9. 
2. Iasechko M., Larin V., Maksiuta D., Ochkurenko O., Samsonov Y., Lyashenko H., Zinchenko A. Influence of ionization source onto macroscopic parameters of the air media in the holes in cops-screensof radio electronic means. APRN Journal of Engineering and Applied Sciences. 2019. № 14 (20). Pp. 3566-3571.

3. Iasechko M., Turinskyi O., Burdin M., Larin V., Gnusov Y., Ikaev D., Borysenko V., Manoylo V. Protection of board radioelectronic equipment from the destructive powerful electromagnetic radiation with the use of natural technologies. IJETER. 2019. № 7 (11). Pp. 542-548.

4. Iasechko M., Sotnikov O., Syrotenko A., Larin V., Iasechko S., Ochkurenko O., Volkov A. Model of Combined Solid Plasma Material for the Protection of Radio-Electronic Means of Optical and Radio Radiation. IJATCSE. 2019. № 8 (4). Pp. 1241-1247.

5. Iasechko M., Sotnikov O., Larin V., Ochkurenko O., Maksiuta D. The model of a medium for creation of electric hermetic screens of the radio electronic means. IJATCSE. 2019. 8(2). Pp. 300-304.

6. Iasechko M., Turinskyi O., Larin V., Prokopenko T., Kolmohorov O., Salash O., Tarshyn V. The Investigation of the Impulse Evolution of the Radio-Frequency and Optical Radiation During the Interaction With the Solid-State Plasma Media On Radioisotope and Hexaferrite Inclusions. IJETER. 2020. 8(4). Pp. 1333-1337. 